\title{
Corela
}

Cognition, représentation, langage

$16-2 \mid 2018$

Vol. $16, n^{\circ} 2$

\section{Les locutions verbales et les constructions à verbe support en français L2}

Alma Bulut and Adel Jebali

\section{CpenEdition}

\section{Journals}

\section{Electronic version}

URL: http://journals.openedition.org/corela/6981

DOI: 10.4000/corela.6981

ISSN: 1638-573X

\section{Publisher}

Cercle linguistique du Centre et de I'Ouest - CerLICO

Electronic reference

Alma Bulut and Adel Jebali, « Les locutions verbales et les constructions à verbe support en français L2 », Corela [Online], 16-2 | 2018, Online since 07 December 2018, connection on 30 April 2019. URL : http://journals.openedition.org/corela/6981; DOI : 10.4000/corela.6981

This text was automatically generated on 30 April 2019

\section{(c) (i) (3) (-)}

Corela - cognition, représentation, langage est mis à disposition selon les termes de la licence Creative Commons Attribution - Pas d'Utilisation Commerciale - Partage dans les Mêmes Conditions 4.0 International. 


\title{
Les locutions verbales et les constructions à verbe support en français L2
}

\author{
Alma Bulut and Adel Jebali
}

\section{Introduction}

1 L'acquisition du vocabulaire d'une langue seconde ou étrangère s'avère un grand défi auquel les apprenants d'une langue nouvelle font face (Kim, 2008; Garza \& Harris, 2016; Hulstijn \& Laufer, 2001). Or, l'acquisition des séquences de mots qui forment des ensembles idiosyncratiques et propres à chaque langue représente pour les apprenants un des plus difficiles aspects d'une L2 (Gonzàlez, 2008, 2007).

2 Le but de la présente étude est d'examiner l'acquisition des verbes complexes (figés ou semi-figés) composés généralement d'un verbe et d'un ou de plusieurs compléments par les apprenants du français langue seconde (désormais FL2). Les verbes complexes sont définis par Gardner \& Davies (2007) comme des séquences de mots formées d'un verbe lexical et d'une ou plusieurs particules (come up with). Les chercheurs qui travaillent dans le cadre théorique du lexique-grammaire (G. Gross, 1996a, 1996b, etc.; M. Gross, 1985, 1993, etc.) divisent généralement ces structures en locutions verbales (a) et en constructions à verbe support (b).

a. avoir froid, prendre garde, jeter la serviette, etc.

b. avoir peur, donner une gifle, faire une promenade, etc.

Pour ce travail, nous adoptons les définitions des locutions verbales (LV) et des constructions à verbe support (CAVS) proposées par G. Gross (1996a). Ainsi, selon cet auteur, une locution verbale est un groupe de mots qui comporte nécessairement un verbe qui doit renfermer une des caractéristiques suivantes : soit son apport sémantique ne peut être déduit à partir des éléments en faisant partie, soit ses compléments ne peuvent être modifiés d'aucune manière en raison de leur rigidité sémantique et syntaxique. Par ailleurs, selon le même auteur, la plus importante propriété d'une CAVS 
est le caractère non figé du déterminant précédant le syntagme nominal de celle-là, même dans les cas où ce déterminant n'est pas réalisé phonétiquement (G. Gross, 1996a).

Plusieurs propriétés définissent les LV et les CAVS et les présenter toutes ici dépasserait largement l'objectif de cet article. Nous renvoyons le lecteur aux analyses des chercheurs qui travaillent dans le cadre théorique du lexique-grammaire, qui nous ont d'ailleurs servi de point de départ pour le présent travail (G. Gross 1996a, 1996b; M. Gross 1993; M. Gross 1988, 1985; Mejri 2008, etc.). Selon ces chercheurs, les LV et les CAVS représentent deux sous-ensembles qui diffèrent significativement l'un de l'autre. Par contre, certaines de ces constructions partagent plusieurs propriétés, ce que nous pouvons constater en observant les exemples (a) et (b). En raison de cela, de nombreux auteurs (Bouffard, 2003, 1995; Gaatone, 1993, 1981; Gougenheim, 1971; etc.) provenant de cadres théoriques différents ont traité les LV et les CAVS en les assimilant les unes aux autres.

5 Comme nous l'avons souligné ci-dessus, l'objectif de cette étude est d'analyser l'acquisition des structures figées en français par les apprenants du FL2. Selon Gonzàlez $(2008,2007)$, l'appropriation de ces structures est très difficile pour ces derniers, car leur construction ne suit pas les règles syntaxiques, lexicales, morphologiques ou sémantiques générales imposées par la grammaire d'une langue donnée. En outre, plusieurs auteurs tels que Gonzàlez (2008, 2007), De Serre (2011) et Detry (2008) soulignent que les constructions verbales complexes ne sont que rarement enseignées aux apprenants d'une L2. Gonzàlez $(2008,2007)$ est d'avis que l'acquisition des séquences figées est primordiale si les apprenants veulent développer des compétences langagières semblables à celles des locuteurs natifs. Comme Dagut \& Laufer (1985) le soulignent, pour que les apprenants réussissent à s'approprier une langue seconde ou étrangère, il faut analyser toutes les difficultés auxquelles ils font face afin de pouvoir les surmonter. Et sachant qu'approximativement $70 \%$ des conversations auxquelles nous participons quotidiennement sont composées de séquences préfabriquées (Wray \& Perkins, 2000) ${ }^{1}$, il semble que l'acquisition des LV et des CAVS soit un des facteurs cruciaux pour la maitrise de la langue que l'on veut acquérir.

6 Prenant appui sur ce dont nous avons discuté ci-dessus, notre objectif est de déterminer si les constructions verbales complexes en français sont évitées par les apprenants de cette langue et, si tel est le cas, nous voudrions être en mesure de déterminer lesquelles des séquences de mots qui font l'objet de notre étude sont les plus souvent évitées ou délaissées par les apprenants: les LV ou les CAVS. Dans le cas où ces constructions seraient évitées par les apprenants, du point de vue pédagogique, l'accent pourrait être mis sur les constructions les plus souvent évitées, ce qui pourrait avoir un grand impact sur l'acquisition du FL2.

\section{Revue de la littérature}

7 Depuis l'apparition de la recherche de Schachter (1974) dans laquelle cette auteure analyse l'évitement des verbes complexes par les apprenants de l'anglais L2, la problématique traitant l'évitement des verbes complexes dans cette langue a fait couler beaucoup d'encre (Liao \& Fukuya, 2004). Par contre, aucune recherche portant sur l'évitement des LV et des CAVS par les apprenants du FL2 n'a été menée jusqu'à présent. Pour cette étude, nous adoptons le terme d'évitement tel que proposé par Laufer \& Eliasson (1993). Selon ces auteurs, l'évitement est l'une des stratégies auxquelles les apprenants ont recours afin de surmonter une difficulté communicative ${ }^{2}$. 
Plusieurs auteurs ont constaté que les verbes complexes sont évités par les apprenants de l'anglais L2 (Dagut \& Laufer, 1985; Liao \& Fukuya, 2004; Siyanova \& Schmitt, 2007; Laufer \& Eliasson, 1993; Hulstijn \& Marchena, 1989; etc.) $)^{3}$. Selon Liao \& Fukuya (2004), Siyanova \& Schmitt (2007), etc., dans la plupart des cas, les apprenants évitent les verbes complexes et utilisent le plus souvent des verbes simples. Siyanova \& Schmitt (2007) pensent que l'évitement est attesté en raison de plusieurs particularités que renferment les verbes complexes. Tout d'abord, ces auteurs considèrent que la raison peut être de nature orthographique puisque les verbes complexes en anglais, à la différence des verbes simples, sont composés de deux unités lexicales. Afin d'acquérir un tel verbe, l'apprenant doit savoir qu'il est composé de plus d'un mot. Si par contre l'apprenant ne sait pas que la dimension sémantique de ce verbe relève de l'union de plusieurs éléments lexicaux, il essayera de décomposer chaque constituant de ce verbe séparément. Selon les mêmes auteurs, l'opacité sémantique des verbes complexes peut jouer un rôle important dans l'évitement de ces derniers ${ }^{4}$.

9 Par ailleurs, les verbes complexes ne suivent pas les règles grammaticales d'une langue donnée : ces unités composées de plusieurs mots sont plutôt stockées dans la mémoire d'où elles sont récupérées comme un tout (Wray \& Perkins, 2000).

Les verbes complexes représentent une partie importante des langues qui en comportent et leur évitement par les apprenants ne semble pas du tout naturel (Gonzàlez, 2007). S'ils sont évités dans les conversations, les apprenants ne feront pas de grands progrès dans leur apprentissage (Gonzàlez, 2007). Par contre, ceux qui utilisent souvent les constructions verbales complexes dans leurs conversations peuvent facilement atteindre un niveau avancé (Gardner \& Davies, 2007; Gonzàlez, 2007). Par ailleurs, les locuteurs natifs utilisent plus souvent les constructions verbales complexes que ne le font les apprenants d'une L2 (Gonzàlez 2007, 2008; Siyanova et Schmitt, 2007; Mel'čuk, 1993; etc.). En outre, Goldberg et Casenhiser (2008) soulignent qu'une langue ne peut être maîtrisée sans l'acquisition des structures phrastiques. Et c'est pour cela que la didactique de ces constructions est aussi importante en L1 qu'en L2, car leur formation est tout à fait arbitraire (Gonzàlez, 2008, 2007)5. Il n'est donc pas surprenant de remarquer que plusieurs auteurs (entre autres Cavalla, 2009b; Detry, 2008; De Serres, 2011; Gonzàlez, 2007, 2008; etc.) soulignent que la didactique des structures phrastiques est aussi nécessaire que la didactique de chaque aspect de la langue que l'on veut acquérir, et ce, dès le début de l'apprentissage.

11 L'objectif principal de cette étude est de vérifier si, à l'instar de l'anglais, l'évitement des verbes composés est aussi observable en français L2 sachant que les auteures telles que Gonzàlez $(2008,2007)$, De Serre (2011), et Detry (2008), entre autres, soulignent que l'acquisition des formes verbales complexes se révèle difficile pour les apprenants d'une L2, car ces structures ne leur sont enseignées que de façon très marginale.

\section{Questions de recherche et hypothèses}

12 Nos questions de recherches découlent de la problématique concernant les constructions verbales complexes en anglais parce que c'est un aspect de la langue française qui a été négligé jusqu'à présent. Notre premier objectif est de vérifier si les constructions verbales complexes en français sont évitées en général par les apprenants du FL2. Afin de répondre à cette question, nous mesurerons le choix des participants entre les verbes 
prédicatifs simples et les constructions verbales complexes (qui en français comportent un verbe lexical et un ou plusieurs compléments). Nous nous attendons à ce que les participants choisissent et produisent le plus souvent les verbes prédicatifs simples. Alors notre première hypothèse est :

Les participants de cette étude choisiront et produiront dans la plupart des cas des verbes prédicatifs simples au détriment des constructions verbales complexes. Ensuite, nous vérifierons les préférences des participants entre les deux structures complexes que nous étudions. Nous croyons que les structures les plus figées seront les mieux réussies. Donc notre deuxième hypothèse est :

Les participants de notre étude choisiront et produiront le plus souvent les LV puisque ces structures verbales complexes sont plus figées que les CAVS et sont stockées telles quelles dans la mémoire.

\section{Méthodologie}

\subsection{Participants}

16 L'étude a été menée à l'université Concordia (Montréal, Canada). De 109 étudiants volontaires qui ont participé à cette étude, 79 étaient apprenants du FL2 des niveaux débutant, intermédiaire et avancé. Comme groupe contrôle, on a recruté 30 participants dont le français est la langue première.

17 En ce qui concerne l'ensemble des participants, 38 d'entre eux sont des hommes (34 \%) et 71 sont des femmes (66\%). L'âge de nos participants varie entre 18 ans et 67 ans (la moyenne est de 25 ans). Les variables de l'âge et du sexe n'ont pas été retenues dans l'analyse des données.

Les participants apprenants du FL2 étaient d'origines différentes. Ce groupe comportait 34 anglophones, 6 hispanophones, 8 sinophones, 7 arabophones et le reste était réparti entre les langues suivantes : le portugais, le hollandais, le perse, le russe, l'hindou, le swahili, l'indonésien, le letton, le grec, le roumain, le bulgare, le hokkien, le malayen et le bengali. Or, ayant remarqué que huit d'entre eux avaient terminé leurs études primaires et secondaires ou même universitaires en français, nous les avons inclus dans le groupe des francophones.

Les 30 participants dont le français est la langue première sont d'origine canadienne ou française. Ils ont tous grandi dans un milieu francophone et ont tous été en contact avec l'anglais. Ils poursuivent leurs études universitaires dans une université anglophone.

\subsection{Matériel}

Pour cette étude, nous avons conçu 4 tâches qui nous ont permis d'analyser la production et la perception des LV et des CAVS par les participants de cette étude, par rapport aux verbes prédicatifs simples ${ }^{6}$ (composés d'un verbe lexical).

21 La première tâche a été un QCM (questionnaire à choix multiples) comportant 15 questions. Chaque exemple proposait aux participants une LV ou une CAVS, telles que définies par G. Gross (1996a, 2008), ainsi qu'un verbe prédicatif simple correspondant sémantiquement à la construction verbale complexe proposée. Nous avons demandé aux 
participants de choisir entre une construction verbale complexe (LV ou CAVS) et un verbe prédicatif simple, qui dans les phrases proposées désignaient une même réalité. L'objectif de cette tâche a été d'amener les participants à choisir la construction qui leur semblait la plus naturelle dans le contexte donné. Par exemple, ils pouvaient choisir soit un verbe prédicatif simple (1) soit une construction verbale complexe (2).

(1) Max a giflé Paul, qui ne peut aucunement comprendre pourquoi il a été giflé par Max.

(2) Max a donné une gifle à Paul, qui ne peut aucunement comprendre pourquoi il a reçu une gifle de Max.

La deuxième tâche comportait 10 questions. L'exercice consistait en la transformation d'un verbe prédicatif simple, comme dans l'exemple (3), en un verbe équivalent ou en une construction verbale complexe. Pour amener les participants à proposer les réponses qui leur semblaient les plus naturelles, nous leur avons suggéré, dans la partie portant sur les explications reliées à cette tâche, qu'ils pouvaient trouver comme synonyme aussi bien des constructions verbales complexes que des verbes prédicatifs simples. Certains des verbes proposés pouvaient être remplacés aussi bien par une LV que par une CAVS. L'objectif de cette tâche était de vérifier si les participants allaient substituer au verbe prédicatif simple une des constructions verbales complexes (une CAVS ou une LV) ou un synonyme sous forme de verbe prédicatif simple.

(3) Max a démissionné7

La troisième tâche se compose d'un QCM contenant 15 questions. Chacune proposait trois énoncés entre lesquels les apprenants pouvaient choisir : une phrase à verbe prédicatif simple, une phrase à verbe complexe (CAVS ou LV) et la proposition « les deux phrases sont équivalentes ", comme le montre l'exemple (4). Cet exercice avait pour but de vérifier si les apprenants remarquaient la synonymie exprimée par les deux sortes de verbes.

(4) 㽞 La fête de Denis se passera dans la maison de sa grand-mère.

啰 La fête de Denis aura lieu dans la maison de sa grand-mère.

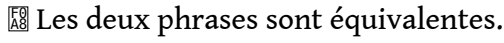

Dans le cadre de la dernière et quatrième tâche, nous avons proposé 11 questions aux participants de cette étude. Pour la compléter, nous avons demandé à ces derniers de traduire de l'anglais vers le français soit un verbe prédicatif simple, soit une LV, soit une CAVS. Un exemple est donné en (5).

(5) The prisoner spilled the beans.

Toutes les tâches proposées aux participants avaient comme but de vérifier la préférence de ces derniers pour un verbe prédicatif simple versus un verbe complexe. Finalement, ces tâches nous ont permis de mesurer et d'analyser certaines stratégies dont se servent les apprenants d'une L2, notamment l'évitement.

Les rencontres avec les participants étaient soit individuelles soit en groupes. Nous leur avons proposé de choisir les réponses qu'ils jugeaient les plus naturelles. Le recrutement a commencé en septembre 2015 et a pris fin en décembre de la même année.

\section{Traitement des données}

Nous nous sommes servis d'un fichier Excel pour le traitement des données. En premier lieu, toutes les données démographiques concernant les participants, telles que leur L1, la langue parlée à la maison, les langues dans lesquelles ils ont été scolarisés aux niveaux 
primaire, secondaire et universitaire, etc. ont été traitées. Cela nous a permis de déterminer les compétences langagières et le niveau de français de chaque participant.

Finalement, nous avons utilisé le programme statistique SPSS pour traiter nos données.

\section{Analyse des données et résultats}

Tout d'abord, nous avons traité les données manquantes afin de déterminer si celles-là différaient en ce qui a trait à la production des constructions verbales complexes et des verbes prédicatifs simples pour les deux groupes de participants. Par ailleurs, nous avons comparé les différences concernant la production et la perception des verbes prédicatifs simples, des CAVS et des LV. Chacun des trois groupes a été traité séparément. Finalement, nous avons traité à part les constructions contenant des fautes de nature sémantique, syntaxique ou orthographique.

\subsection{Analyse des données manquantes}

Étant donné que nous avons remarqué que plusieurs participants n'avaient soumis aucune réponse dans le cadre des tâches $(2)^{8}$ et $(4)^{9}$, notre premier objectif a été de vérifier si le groupe d'apprenants du FL2 omettait certaines réponses au même titre que le groupe de locuteurs natifs du français. Le programme SPSS a traité ces omissions comme données manquantes. Dans le cadre de cette étude, les locuteurs natifs du français omettaient des réponses dans $4,75 \%$ des cas tandis que les apprenants du français le faisaient dans $14,86 \%$ des cas.

\begin{tabular}{|l|l|l|l|l|}
\hline & Francophone & $\mathbf{N}$ & Moyenne & Écart type \\
\hline \multirow{2}{*}{ Nombre de réponses manquantes } & Francophone & 38 &, 0475 &, 07027 \\
\cline { 2 - 6 } & Non-francophone & 71 &, 1486 &, 10850 \\
\hline
\end{tabular}

Tableau 1 : Distribution des données manquantes

31 Le test $\mathrm{U}$ de Mann-Whitney démontre que le ratio des réponses manquantes entre le groupe francophone et le groupe non francophone n'est pas identique.

\begin{tabular}{|l|l|l|l|}
\hline Hypothèse nulle & Test & Sig. & Décision \\
\hline $\begin{array}{l}\text { La distribution du ratio des réponses } \\
\text { manquantes est identique pour les deux } \\
\text { groupes. }\end{array}$ & $\begin{array}{l}\text { Test U de Mann-Whitney } \\
\text { d'échantillons indépendants. }\end{array}$ &, 000 & $\begin{array}{l}\text { Rejeter } \\
\text { l'hypothèse } \\
\text { nulle. }\end{array}$ \\
\hline
\end{tabular}

Tableau 2 : Test $\mathrm{U}$ de Mann-Whitney pour la distribution des données manquantes

Ces données nous permettent de conclure que la différence entre les deux groupes est significative en ce qui a trait aux données manquantes $(p=, 000)$. Les locuteurs natifs du français fournissaient plus de réponses que ne le faisaient les apprenants de cette langue. 


\subsection{Analyse du choix des verbes simples vs les LV} prédicatifs simples. Les tâches $(1)^{10}$ et $(3)^{11}$ sont celles que nous avons analysées dans ce but. L'analyse des données démontre que les apprenants choisissent les verbes simples au détriment des LV dans 59,38 \% des cas. Par contre, les francophones choisissent les premiers verbes versus les LV en moyenne à $51,39 \%$.

\begin{tabular}{|l|l|l|l|l|}
\hline & Francophone & $\mathbf{N}$ & Moyenne & Écart type \\
\hline \multirow{2}{*}{ Ratio verbe simple vs LV } & Francophone & 38 &, 5139 &, 22075 \\
\cline { 2 - 5 } & Non-francophone & 71 &, 5938 &, 13759 \\
\hline
\end{tabular}

Tableau 3 : Distribution des verbes simples vs les LV

Encore une fois, le test statistique U de Mann-Whitney a été utilisé pour cette analyse. Le ratio des verbes simples vs les LV est identique pour les deux groupes.

\begin{tabular}{|l|l|l|l|}
\hline Hypothèse nulle & Test & Sig. & Décision \\
\hline $\begin{array}{l}\text { La distribution du ratio verbe simple } \\
\text { vs LV est identique pour les deux } \\
\text { groupes. }\end{array}$ & $\begin{array}{l}\text { Test U de Mann-Whitney } \\
\text { d'échantillons indépendants. }\end{array}$ & $\begin{array}{l}\text { Retenir } \\
\text { l'hypothèse } \\
\text { nulle. }\end{array}$ \\
\hline
\end{tabular}

Tableau 4 : Test U de Mann-Whitney pour la distribution des verbes simples vs les LV

Ce test met en évidence que la différence entre les apprenants et les locuteurs natifs de notre étude n'est pas significative $(p=0,148)$. Les deux groupes choisissaient de la même manière les verbes simples au détriment des LV.

\subsection{Analyse du choix des verbes simples vs les CAVS}

Pour tester le choix des participants entre les verbes simples et les CAVS, encore une fois, les réponses des tâches (1) et (3) ont été analysées. Les apprenants choisissent les verbes simples par rapport aux CAVS en moyenne à $44,69 \%$ contrairement aux locuteurs natifs qui les choisissaient dans $40,19 \%$ des cas.

\begin{tabular}{|l|l|l|l|l|}
\hline & Francophone & N & Moyenne & Écart type \\
\hline \multirow{2}{*}{ Ratio verbe simple vs CAVS } & Francophone & 38 &, 4019 &, 13800 \\
\cline { 2 - 5 } & Non-francophone & 71 &, 4469 &, 13374 \\
\hline
\end{tabular}

Tableau 5 : Distribution des verbes simples vs les CAVS 
Similairement aux résultats concernant le choix entre les LV et les verbes simples, les résultats du test statistique $U$ de Mann-Whitney $(\mathrm{p}=0,145)$ démontrent que la différence entre les apprenants et les locuteurs natifs n'est pas significative en ce qui concerne les choix entre les verbes simples et les CAVS.

\begin{tabular}{|l|l|l|l|}
\hline Hypothèse nulle & Test & Sig. & Décision \\
\hline $\begin{array}{l}\text { La distribution du ratio verbe simple vs } \\
\text { CAVS est identique pour les deux } \\
\text { groupes. }\end{array}$ & $\begin{array}{l}\text { Test U de Mann-Whitney } \\
\text { d'échantillons indépendants. }\end{array}$ & $\begin{array}{l}\text { Retenir } \\
\text { l'hypothèse } \\
\text { nulle. }\end{array}$ \\
\hline
\end{tabular}

Tableau 6 : Test U de Mann-Whitney pour la distribution des verbes simples vs les CAVS

Selon ces données, les deux groupes choisissaient à la même fréquence les verbes simples et les CAVS.

\subsection{Production des verbes simples vs les constructions verbales complexes}

Afin d'analyser la production des constructions verbales complexes par rapport aux verbes prédicatifs simples, nous avons analysé les données des tâches (2) (qui consistait en la transformation d'un verbe prédicatif simple) et (4) (qui consistait en la traduction d'une construction verbale complexe du français vers l'anglais). Dans notre échantillon, les apprenants transformaient un verbe simple ou complexe en un verbe simple en moyenne à 65,37 \% tandis que les locuteurs natifs le faisaient dans 59, $20 \%$ des cas.

\begin{tabular}{|l|l|l|l|l|}
\hline & Francophone & N & Moyenne & Écart type \\
\hline \multirow{2}{*}{ Ratio choix de verbe simple } & Francophone & 38 &, 5920 &, 13316 \\
\cline { 2 - 5 } & Non-francophone & 71 &, 6537 &, 15587 \\
\hline
\end{tabular}

Tableau 7 : Production des verbes simples

Le test statistique démontre que la différence entre les deux groupes est significative $(p=, 009)$.

\begin{tabular}{|l|l|l|l|}
\hline Hypothèse nulle & Test & Sig. & Décision \\
\hline $\begin{array}{l}\text { La distribution du ratio choix de verbe } \\
\text { simple est identique pour les deux } \\
\text { groupes. }\end{array}$ & $\begin{array}{l}\text { Test U de Mann-Whitney } \\
\text { d'échantillons indépendants. }\end{array}$ &, 009 & $\begin{array}{l}\text { Rejeter } \\
\text { l'hypothèse } \\
\text { nulle. }\end{array}$ \\
\hline
\end{tabular}

Tableau 8 : Test $\mathrm{U}$ de Mann-Whitney pour la production des verbes simples 
41 Nous pouvons conclure que les apprenants de notre échantillon traduisaient ou reformulaient les verbes proposés en un verbe simple au détriment des verbes complexes plus souvent que ne le faisaient les locuteurs natifs.

\subsection{Production des LV vs les verbes simples}

Notre objectif a été également de tester la production des LV par rapport aux verbes prédicatifs simples. Les apprenants du FL2, dans notre échantillon, produisaient des LV versus les verbes simples en moyenne à $6,40 \%$ tandis que les locuteurs natifs faisaient ce choix dans $14,67 \%$ des cas.

\begin{tabular}{|l|l|l|l|l|}
\hline & Francophone & $\mathbf{N}$ & Moyenne & Écart type \\
\hline \multirow{2}{*}{ Ratio choix de LV } & Francophone & 38 &, 1467 &, 09807 \\
\cline { 2 - 5 } & Non-francophone & 71 &, 0640 &, 06725 \\
\hline
\end{tabular}

Tableau 9: Production des LV vs les verbes simples

La différence entre les deux groupes est significative $(p=, 000)$

\begin{tabular}{|l|l|l|l|}
\hline Hypothèse nulle & Test & Sig. & Décision \\
\hline $\begin{array}{l}\text { La distribution du ratio choix de LV } \\
\text { est identique pour les deux groupes. }\end{array}$ & $\begin{array}{l}\text { Test U de Mann-Whitney } \\
\text { d'échantillons indépendants. }\end{array}$ & $\begin{array}{l}\text { Rejeter } \\
\text { l'hypothèse } \\
\text { nulle. }\end{array}$ \\
\hline
\end{tabular}

Tableau 10 : Test U de Mann-Whitney pour la production des LV vs les verbes simples

Dans notre échantillon, les locuteurs natifs du français produisaient plus de LV que ne le faisaient les apprenants de cette langue.

\subsection{Production des CAVS vs les verbes simples}

Nous avons également analysé les données de cette étude dans le but de vérifier à quelle fréquence les participants produisaient les CAVS par rapport aux verbes prédicatifs simples. Les données descriptives démontrent que les apprenants produisent ces constructions complexes en moyenne à $20,64 \%$ alors que les locuteurs natifs le font dans $23,04 \%$ des cas.

\begin{tabular}{|l|l|l|l|l|}
\hline & Francophone & $\mathbf{N}$ & Moyenne & Écart type \\
\hline \multirow{2}{*}{ Ratio choix des CAVS } & Francophone & 38 &, 2304 &, 10303 \\
\cline { 2 - 5 } & Non-francophone & 71 &, 2064 &, 12908 \\
\hline
\end{tabular}

Tableau 11 : Production des CAVS vs les verbes simples 
Le ratio du choix des CAVS versus les verbes simples est identique pour les deux groupes de participants, selon le test U Mann-Whitney.

\begin{tabular}{|l|l|l|l|}
\hline Hypothèse nulle & Test & Sig. & Décision \\
\hline $\begin{array}{l}\text { La distribution du ratio choix de CAVS } \\
\text { est identique pour les deux groupes. }\end{array}$ & $\begin{array}{l}\text { Test U de Mann-Whitney } \\
\text { d'échantillons indépendants. }\end{array}$ &, 141 & $\begin{array}{l}\text { Retenir } \\
\text { l'hypothèse } \\
\text { nulle. }\end{array}$ \\
\hline
\end{tabular}

Tableau 12 : Test U de Mann-Whitney pour la production des CAVS vs les verbes simples

La différence entre les deux groupes n'est pas significative $(p=, 141)$.

\subsection{Analyse de la grammaticalité des LV produites par les participants}

48 Les données des tâches (2) et (4) ont été analysées pour tester la grammaticalité des constructions verbales produites par les participants de cette étude. Nous avons jugé comme agrammaticale toute construction verbale comportant des fautes de nature syntaxique, sémantique, lexicale ou orthographique.

Plusieurs participants apprenants du français n'ont produit aucune construction verbale complexe dans le cadre de cette étude. Par contre, les locuteurs natifs étaient plus productifs à cet égard. Par exemple, 36 sur 38 de ces derniers ont produit au moins une LV tandis que seulement 41 sur 71 apprenants du français en ont fait autant. Pour notre analyse, il est nécessaire de souligner que le programme SPSS a traité comme réussite à $100 \%$ des cas où toutes les LV produites ne comportent aucune faute. Il importe de préciser que tel est le cas même si le participant n'a produit qu'une seule construction verbale grammaticale. Si, par contre, deux constructions verbales ont été produites dont une est correcte et la deuxième comporte des fautes, le programme SPSS a traité de tels cas comme réussite à $50 \%$.

Les données de notre échantillon démontrent que les apprenants produisaient des LV grammaticales dans 77,98 \% des cas. Par contre, les locuteurs natifs du français le faisaient en moyenne à $96,06 \%$.

\begin{tabular}{|c|c|c|c|c|}
\hline & Francophone & $\mathbf{N}$ & $\begin{array}{l}\text { Moyenne des bonnes } \\
\text { réponses }\end{array}$ & $\begin{array}{l}\text { Moyenne des } \\
\text { erreurs }\end{array}$ \\
\hline \multirow{2}{*}{$\begin{array}{l}\text { Ratio des } \\
\text { grammaticales }\end{array}$} & Francophone & 36 & ,9606 &, 0394 \\
\hline & $\begin{array}{l}\text { Non- } \\
\text { francophone }\end{array}$ & 42 & ,7798 &, 2202 \\
\hline
\end{tabular}

Tableau 13 : Distribution de la production des LV grammaticales

51 Le test U de Mann-Whitney démontre que la différence entre les deux groupes est significative $(p=, 011)$. 


\begin{tabular}{|l|l|l|l|}
\hline Hypothèse nulle & Test & Sig. & Décision \\
\hline $\begin{array}{l}\text { La distribution du ratio de la } \\
\text { production des LV est identique pour } \\
\text { les deux groupes. }\end{array}$ & $\begin{array}{l}\text { Test U de Mann-Whitney } \\
\text { d'échantillons indépendants. }\end{array}$ & $\begin{array}{l}\text { Rejeter } \\
\text { l'hypothèse } \\
\text { nulle. }\end{array}$ \\
\hline
\end{tabular}
participants de cette étude. De 38 locuteurs natifs du français, encore une fois, seulement deux n'ont produit aucune CAVS tandis que du côté des apprenants, sept n'ont fourni aucune réponse. Le premier groupe réussissait la production des CAVS dans 95,65\% des cas tandis que le deuxième en faisait autant en moyenne à 73,68 \%.

\begin{tabular}{|l|l|l|l|l|}
\hline & Francophone & $\mathbf{N}$ & $\begin{array}{l}\text { Moyenne des bonnes } \\
\text { réponses }\end{array}$ & $\begin{array}{l}\text { Moyenne des } \\
\text { erreurs }\end{array}$ \\
\hline \multirow{2}{*}{$\begin{array}{l}\text { Ratio des CAVS } \\
\text { grammaticales }\end{array}$} & Francophone & 36 &, 9565 &, 0435 \\
\cline { 2 - 6 } & $\begin{array}{l}\text { Non- } \\
\text { francophone }\end{array}$ & 64 &, 7368 &, 2632 \\
\hline
\end{tabular}

Tableau 15 : Distribution de la production des CAVS grammaticales

La différence entre les deux groupes est significative $(p=, 000)$.

\begin{tabular}{|l|l|l|l|}
\hline Hypothèse nulle & Test & Sig. & Décision \\
\hline $\begin{array}{l}\text { La distribution du ratio de la production } \\
\text { des CAVS est identique pour les deux } \\
\text { groupes. }\end{array}$ & $\begin{array}{l}\text { Test U de Mann-Whitney } \\
\text { d'échantillons indépendants. }\end{array}$ & $\begin{array}{l}\text { Rejeter } \\
\text { l'hypothèse } \\
\text { nulle. }\end{array}$ \\
\hline
\end{tabular}

Tableau 16 : Test U de Mann-Whitney pour la production des CAVS grammaticales

Le test U de Mann-Whitney met en lumière que les francophones réussissaient mieux la reformulation ainsi que la traduction de l'anglais vers le français des CAVS que le groupe d'apprenants. 


\subsection{Analyse de l'équivalence entre les constructions verbales complexes et les verbes simples}

Dans le cadre de la troisième tâche, les participants pouvaient choisir entre une construction verbale complexe, un verbe prédicatif simple et la proposition que les deux sortes de verbe sont équivalents. Les participants dont le français est la langue première choisissaient la proposition d'équivalence en moyenne à $47,19 \%$ tandis que les apprenants du français le faisaient dans $34,74 \%$ des cas.

\begin{tabular}{|l|l|l|l|l|}
\hline & Francophone & $\mathbf{N}$ & Moyenne & Écart type \\
\hline \multirow{2}{*}{ Ratio deux propositions sont équivalentes } & Francophone & 38 &, 4719 &, 21886 \\
\cline { 2 - 6 } & Non-francophone & 71 &, 3474 &, 18202 \\
\hline
\end{tabular}

Tableau 19 : Distribution de la proposition «les deux phrases sont équivalentes »

Le test $\mathrm{U}$ de Mann-Whitney met en lumière que la différence entre les deux groupes est significative $(p=, 004)$

\begin{tabular}{|l|l|l|l|}
\hline Hypothèse nulle & Test & Sig. & Décision \\
\hline $\begin{array}{l}\text { La distribution de ratio deux phrases } \\
\text { sont équivalentes est identique pour les } \\
\text { deux groupes. }\end{array}$ & $\begin{array}{l}\text { Test U de Mann-Whitney } \\
\text { d'échantillons } \\
\text { indépendants. }\end{array}$ &, 004 & $\begin{array}{l}\text { Rejeter } \\
\text { l'hypothèse } \\
\text { nulle. }\end{array}$ \\
\hline
\end{tabular}

Tableau 18 : Test $\mathrm{U}$ de Mann-Whitney pour la distribution de la proposition « les deux phrases sont équivalentes »

Suite à ces analyses, nous pouvons conclure que, dans notre échantillon, les locuteurs natifs du français s'aperçoivent plus souvent de la synonymie des verbes proposés que ne le font les apprenants de cette langue.

\section{Interprétation des résultats}

L'objectif de la présente étude étant d'examiner l'acquisition des structures verbales complexes par les apprenants du FL2, nous nous sommes penchés sur l'analyse des données afin de tester nos hypothèses proposées dans la section 3. de ce travail. Tout d'abord, notre objectif est d'analyser si, à l'instar des apprenants de l'anglais L2, les apprenants du FL2 évitent les constructions verbales complexes en français. Nous avons émis l'hypothèse que les apprenants du FL2 éviteraient en général les constructions verbales complexes en français.

61 Ensuite, notre objectif est d'analyser laquelle des deux structures verbales mises par les procédés grammaticaux à la disposition des locuteurs du français est la plus productive : les CAVS ou les LV. Nous proposons que les constructions les plus figées, que sont les LV selon les chercheurs travaillant dans le cadre du lexique-grammaire, soient mieux 
réussies que les CAVS, qui, de leur côté, représentent généralement une structure syntaxique moins figée.

62 Les résultats des tests statistiques nous ont menés parfois à des constats surprenants. Dans le cadre des tâches (1) et (3), où les CAVS étaient aussi présentes que les LV, nous avons demandé aux participants de choisir la structure qui leur semblait la plus naturelle. Dans le cas où les participants faisaient face à des CAVS, la différence entre les apprenants du FL2 et le groupe contrôle n'était pas significative. Cela signifie que les deux groupes choisissaient à la même fréquence les CAVS au détriment des verbes prédicatifs simples.

63 Les résultats des tests statistiques démontrent que le choix des participants en ce qui concerne les exemples proposant des LV et des verbes simples exprimant une même réalité est similaire à celui déjà mentionné, malgré le fait que l'écart entre les deux groupes est plus significatif dans ce cas. À la lumière de ces observations, nous pouvons conclure que les apprenants du FL2 choisissaient à la même fréquence que les locuteurs natifs $d u$ français les constructions verbales complexes au détriment des verbes prédicatifs simples.

64 En revanche, dans le cas où les participants devaient soit transformer un verbe prédicatif simple en une construction verbale complexe (tâche 2) soit traduire de l'anglais vers le français un verbe complexe (tâche 4), les résultats sont tout à fait divergents. À la différence des tâches (1) et (3) où les participants n'avaient qu'à choisir entre ce qui leur avait été proposé, dans le cadre des deux tâches (2) et (4), ils devaient produire des constructions verbales complexes, ce qui exigeait des connaissances préalables dans ce domaine. En ce qui concerne la production des CAVS, dans notre échantillon, il n'y avait pas de différence significative entre les apprenants du FL2 et les locuteurs natifs. Par contre, les constats sont tout à fait différents dans le cas où les participants faisaient face aux LV. En effet, dans notre échantillon, les locuteurs natifs du français en produisaient beaucoup plus que ne le faisaient les apprenants de cette langue. Ces derniers choisissaient dans la plupart des cas d'omettre la réponse. Dans les cas où ils fournissaient une réponse, ils optaient plutôt pour la reformulation en français ou la traduction de l'anglais vers le français en utilisant un verbe prédicatif simple au détriment des LV. Notons que les cas où les participants évitaient d'offrir une réponse ont été traités par le programme SPSS comme données manquantes et celles-ci sont beaucoup plus fréquentes pour les apprenants de notre échantillon que pour le groupe contrôle.

Suite aux analyses présentées ci-dessus, nous nous sommes penchés sur la grammaticalité des constructions verbales complexes produites par les deux groupes de notre étude. Comme nous l'avons déjà mentionné, les apprenants du FL2 produisaient moins de structures verbales complexes que ne le faisait le groupe contrôle. En outre, le premier groupe a produit plus de constructions verbales agrammaticales que le deuxième groupe, et ce, aussi bien dans les cas où ils proposaient des LV que dans ceux proposant des CAVS. Nous pouvons conclure que les locuteurs natifs produisaient en général plus de structures verbales complexes grammaticales que ne le faisaient les apprenants de notre échantillon.

D'autres résultats ayant rapport aux différences caractérisant les locuteurs natifs du français et les apprenants du FL2 font surface. En effet, dans le cadre de la tâche (3), nous avons demandé aux participants de choisir un des trois énoncés: un verbe prédicatif simple, une construction verbale complexe synonyme de ce premier et la proposition que les deux premiers sont équivalents. Encore une fois, les locuteurs natifs de notre étude 
ont démontré une meilleure maîtrise des constructions verbales complexes étant donné qu'ils sont plus nombreux à se rendre compte de la synonymie des verbes suggérés.

\section{Discussion}

Quelques-uns de nos résultats nous amènent à penser que les participants non francophones de notre étude maitrisaient certains aspects des structures verbales figées en français, ce qui va à l'encontre de notre première hypothèse selon laquelle les participants non francophones éviteraient en général les LV et les CAVS. Cela est particulièrement observable dans les tâches (1) et (3) où le but était de tester la perception des structures en question. Dans le cadre de ces tâches, les participants n'avaient qu'à choisir un des énoncés qui leur avaient été proposés. Nous proposons que la manière dont ces tâches avaient été conçues ait grandement facilité la perception aussi bien que la compréhension des structures verbales complexes. Cette observation est particulièrement intéressante dans les cas où les participants devaient choisir entre un verbe prédicatif simple et une CAVS. En effet, dans la grande majorité des exemples proposés dans notre étude, le lien de synonymie entre les verbes prédicatifs simples et les CAVS était évident (par exemple : faire une promenade - se promener, donner des conseils conseiller, pousser un hurlement - hurler, etc.). Nous suggérons que les participants non francophones n'aient aucune difficulté à s'apercevoir de la synonymie des deux verbes, qui, d'ailleurs, leur avaient été proposés dans des contextes identiques.

Par ailleurs, en ce qui a trait à la perception concernant les exemples qui contenaient des LV, il n'y avait non plus de différence significative entre les deux groupes de notre étude. Cela veut dire que les participants non francophones choisissaient à la même fréquence les LV que les locuteurs natifs du français de cette étude ${ }^{12}$. Or, dans ce cas-là, ces premiers ne pouvaient pas se fier à la synonymie entre les énoncés proposés vu que, en général, les LV et les verbes prédicatifs simples leur correspondant sémantiquement ne comportent pas de mots du même champ lexical (par exemple : passer quelqu'un à tabac signifie frapper violemment), ce qui est par contre souvent le cas des CAVS. Est-il donc possible que les participants apprenants du FL2 maîtrisaient les LV aussi bien que les participants francophones? Pas nécessairement. Comme Detry (2008), Irujo (1986) et Gonzales (2008) le soulignent, l'apprentissage des constructions verbales complexes d'une L2 peut être facilité si l'apprenant réussit à trouver des constructions complexes semblables dans sa L1. Par exemple, certaines LV comportent beaucoup de similarités d'une langue à l'autre (par ex. : aller le nez au vent se dit en serbo-croate dignuti nos (lever le nez). Les deux LV comportent le SN nez et l'image métaphorique que l'on se crée à partir des éléments syntaxiques formant chacune des deux suites des mots permet aux nouveaux apprenants d'en saisir le sens). En outre, certaines LV sont identiques d'une langue à l'autre (briser la glace (français), rompre el hielo (espagnol), brake the ice (anglais), razbiti led (serbo-croate), das Ice brechen (allemand), etc). Detry (2008) souligne que l'apprenant pourrait facilement trouver le sens d'une construction figée s'il réussit à trouver un lien entre les éléments syntaxiques la composant et l'image métaphorique que ses éléments créent comme un ensemble.

De son côté, Gonzales (2008), bien qu'elle souligne que les structures figées sont rarement enseignées aux apprenants d'une $\mathrm{L} 2^{13}$, met en évidence que quelques LV sont bien présentes dans des manuels destinés aux apprenants, sans pourtant être enseignées de manière adéquate. Nous suggérons qu'il soit possible que certains participants de notre 
étude aient lu des textes comportant des LV et que ces connaissances acquises préalablement ont fait surface lorsqu'ils étaient confrontés à des structures déjà vues.

Les observations dont nous avons discuté ci-dessus sont encore plus intéressantes lorsque nous analysons les résultats des tests statistiques concernant les tâches (2) et (4). En effet, dans le cadre de ces tâches, les participants devaient produire des CAVS et des LV. Et dans notre échantillon, le groupe francophone a réussi beaucoup mieux ces deux tâches-là que le groupe non francophone, ce qui confirme partiellement notre première hypothèse. C'est cette facette de notre échantillon qui met en lumière la divergence entre les deux groupes de notre étude. Et c'est exactement pour cela que plusieurs chercheurs (entre autres: Siyanova et Schmitt, 2007; Gonzales, 2008, 2007; Cavalla, 2009a, 2009b; etc.) préconisent que la didactique des structures verbales figées soit intégrée dans les classes d'enseignement, et ce, dès le début de l'apprentissage d'une L2. D'ailleurs, Cavalla (2009b) met en exergue que cela est encore plus important en raison de la complexité que représentent les constructions complexes ${ }^{14}$ pour les apprenants d'une L2, vu que leur maîtrise exige une parfaite connaissance des domaines morphologique, syntaxique, lexical et sémantique. Encore selon Cavalla (2009b), les apprenants d'une langue ont tendance à utiliser des combinaisons de mots qui ne semblent pas naturelles pour les locuteurs natifs d'une langue et cela est particulièrement observable avec les CAVS, souligne cette auteure, appuyant ainsi l'idée de Gonzales (2008) que les CAVS sont les structures figées les plus délaissées dans l'enseignement d'une L2.

71 Si les CAVS représentent une difficulté pour les apprenants d'une L2, les LV ne font aucune exception à cet égard. En effet, Gonzales (2008) propose que les apprenants d'une L2 aient des difficultés dès le début de leur apprentissage, car, étant refoulées et presque abandonnées dans les cours d'enseignement, en raison des croyances linguistiques selon lesquelles l'utilisation des expressions idiomatiques dévalorise et même abaisse le niveau de langue utilisée, ces structures complexes représentent une entrave signifiante pour les apprenants d'une L2. Toujours selon Gonzales (2008), le manque de maîtrise des LV d'une L2 peut entraver à jamais l'accès aux niveaux supérieurs de la langue cible. En outre, Geneviève (1998) souligne que « le vrai français ", qui possède cette faculté extraordinaire d'exprimer en quelques tournures phraséologiques des contextes complexes, n'est jamais enseigné aux apprenants des L2. Et sachant que la maitrise des structures idiomatiques, dont la plupart des LV font partie, requiert une excellente maîtrise des domaines syntaxique, sémantique, morphologique ainsi que pragmatique (De Serre, 2011), il est très important que la phraséologie soit adéquatement introduite et expliquée dans chaque niveau d'enseignement des L2.

Dans la présente étude, le fait que les apprenants du FL2 repèrent les constructions verbales complexes à la même fréquence que les locuteurs natifs du français, mais que la production de ces séquences de mots s'avère plus difficile pour le premier groupe de participants, va de pair avec l'observation de Gonzales (2008) selon laquelle les manuels destinés aux apprenants des L2 ciblent la compréhension et non pas la production des constructions figées. Cette observation est également appuyée par les résultats de la recherche de Flege (1993), qui a remarqué que les apprenants de l'anglais repèrent les voyelles dans cette langue à la même fréquence que les anglophones, mais qu'il y avait une différence significative entre les deux groupes de participants dans les tâches exigeant la production de ces sons. 


\section{Conclusion}

73 L'objectif principal de la présente étude a été d'étudier et d'analyser la perception et la production des constructions verbales complexes par les apprenants du FL2. En nous appuyant sur les travaux proposés par les chercheurs du cadre théorique du lexiquegrammaire, nous avons divisé les séquences de mots étudiées en deux groupes distinctifs : les LV et les CAVS. Nous avons démontré, dans le cadre de ce travail, qu'il est nécessaire d'introduire l'enseignement de ces séquences de mots aux apprenants du FL2 afin que ces derniers, à l'instar des locuteurs natifs du français, apprennent à maîtriser la production des expressions en question. Par contre, nous avons constaté que les participants non francophones repéraient à la même fréquence les LV et les CAVS que les locuteurs natifs de notre étude. Nous avons démontré que l'acquisition des structures verbales complexes suit un ordre chronologique particulier étant donné que la perception précède la production lors de l'acquisition des L2.

Il est également nécessaire de souligner que la conception des tâches proposées aux participants ait pu influencer les résultats de notre étude. Tout d'abord, comme le proposent Gonzales (2008) et Siyanova et Schmitt (2007), les constructions verbales complexes sont beaucoup plus fréquentes dans les conversations orales que dans la langue écrite. Il est alors fort possible que les résultats aient pu différer si nous avions proposé aux participants les tests oraux et non pas écrits. En outre, étant donné que certaines constructions verbales complexes sont plus fréquentes que les autres (Siyanova et Schmitt, 2007), il est possible que de telles constructions puissent influencer le choix des participants. Nous ne savons pas lesquelles des constructions verbales complexes incluses dans notre étude sont plus fréquentes que les autres. Il est aussi important de remarquer que le choix des exemples des LV et des CAVS dans cette étude aurait pu jouer un rôle important. Autrement dit, si nous avions utilisé d'autres exemples des LV et des CAVS, les résultats auraient pu différer.

Et finalement, il importe de préciser que nous n'avons pas examiné les procédés grammaticaux servant à la création des verbes dans les L1 de nos participants apprenants du FL2 et nous ne savons pas si certains d'entre eux évitaient tout simplement les constructions verbales complexes en raison de leur absence dans leur L1.

En conclusion générale, nous aimerions ajouter que, en ce qui concerne le groupe d'apprenants du FL2 de notre étude, dans les exemples proposés, il y avait un écart significatif entre les LV et les CAVS, et ce, aussi bien dans les tâches de production que dans les tâches de perception. En effet, comme nous l'avons déjà mentionné ci-dessus, ce groupe produisait plus souvent des CAVS (en moyenne à 20,64 \%) que des LV (en moyenne à 6,40\%) au détriment des verbes prédicatifs simples. Les mêmes constats sont observables dans les tâches de perception où les verbes prédicatifs simples, dans les exemples étant en synonymie avec des LV, étaient choisis en moyenne à 59,38 \%, tandis que ceux étant en synonymie avec des CAVS ont été repérés dans 44,69\% des cas. Découle de cette observation que les CAVS sont mieux réussies que les LV par les participants non francophones de notre échantillon. Ces résultats rejettent notre deuxième hypothèse selon laquelle les apprenants du FL2 réussiraient mieux les constructions les plus figées en raison de leur rigidité syntaxique. Il importe de mettre en lumière que, dans le cadre de cette étude, nous ne serons pas en mesure de proposer une explication à cette observation. Or, des recherches supplémentaires pourraient lever le voile sur ce 
phénomène afin de déterminer si la rigidité syntaxique du syntagme nominal des LV ou bien la dimension idiomatique de celles-là y jouent un rôle important. Et pour le faire, il faudrait tester, dans le cadre d'une même étude, des verbes complexes comportant uniquement des LV dont le sens est compositionnel et des CAVS, aux structures syntaxiques quasi identiques. De cette façon, on écarterait les différences sémantiques qui décalent les LV et les CAVS et on pourrait tester si la rigidité syntaxique joue un rôle important en ce qui concerne la perception, mais aussi la production des structures verbales figées ou semi-figées en français.

\section{BIBLIOGRAPHY}

BOUFFARD, Paula (1995). Le traitement formel des composés verbaux, Mémoire de maîtrise, Université du Québec à Montréal, Montréal.

BOUFFARD, Paula (2003). Les locutions verbales en français : traitement unifié dans une approche lexicaliste, Thèse de doctorat, Université du Québec à Montréal, Montréal.

CAVAlla, C. (2009a). Propositions didactiques pour l'enseignement d'éléments phraséologiques en FLE. Enseigner les structures langagières en FLE, Dan Van Raemdonck et Marie-Eve Damar. Gramm-

FLE.

CAVALLA, C. (2009b). La phraséologie en classe de FLE. Les langues modernes, 1, 2009.

DAgUt, M., \& LAUfER, B. (1985). Avoidance of Phrasal Verbs-A Case for Contrastive Analysis. Studies in Second Language Acquisition, 7(01), 73-80. doi:10.1017/s0272263100005167

DE SERRES, L. (2011). Tendances en enseignement des expressions idiomatiques en langue seconde : de la théorie à la pédagogie. The Canadian Journal of Applied Linguistics, 14(2), 129.

DETRY, F. (2008). Pourquoi les murs auraient-ils des oreilles? Vers un apprentissage par l'image des expressions idiomatiques en langue étrangère. Synergies (Espagne), 1, 205-218.

FLEGE, J. E. (1993). Production and perception of a novel, second-language phonetic contrast. The Journal of the Acoustical Society of America, 93(3), 1589-1608. doi:10.1121/1.406818

GAATONE, D. (1993). Les locutions verbales et les deux passifs du français. Langages, 27(109), 37-52. doi:10.3406/lgge.1993.1090

GARDNER, D., \& DAVIES, M. (2007). Pointing out frequent phrasal verbs: A Corpus-Based Analysis. TESOL Quarterly, 41(2), 339-359. doi:10.1002/j.1545-7249.2007.tb00062.x

GARZA, B., \& HARRIS, R. J. (2016). Acquiring foreign language vocabulary through meaningful linguistic context: Where is the limit to vocabulary learning?. Journal of Psycholinguistic Research. doi:10.1007/s10936-016-9444-0 GENEVIÈVE. (1998). Merde!: the real French you were never taught at school. Simon and Schuster. GIRY-SCHNEIDER, J. (1991a). Relation entre le sens des noms et leur structure prédicative. Revue québécoise de linguistique, 20(1), 99-124. 
GIRY-SCHNEIDER, J. (1991b). L'article zéro dans le lexique-grammaire des noms prédicatifs. Langages , (102), 23-35. doi : 10.3406/lgge.1991.1597

GOLDBERG, A. E., \& CASENHISER, D. (2008). Construction Learning and Second Language Acquisition. In P. Robinson \& N. Ellis (Eds.), Handbook of Cognitive Linguistics and Second Language Acquisition. 197-215. New York : Routledge.

GONZÀLEZ, Rey, I. (2007). La didactique du français idiomatique. Fernelmont : EME.

GONZÀLEZ, Rey, I. (2008). La phraséodidactique en action : les expressions figées comme objet d'enseignement. In La culture de l'autre : l'enseignement des langues à l'Université, École Normale Supérieure Lettres et Sciences Humaines.

GONZÀLEZ, Rey, I. (2014). Outils et méthode d'apprentissage en phraséodidactique : Essai de didactique. EME éditions. Éditions modulaires européennes.

goUgenheim, G. (1971). Une catégorie lexico-grammaticale : les locutions verbales. Études de linguistique appliquée, 2, 56.

GROSS, G. (1996a). Les expressions figées en français : noms composés et autres locutions. Éditions Ophrys.

GROSS, G. (1996b). Prédicats nominaux et compatibilité aspectuelle. Langages, 30(121), 54-72. doi ; 10.3406/lgge.1996.1740

GROSS, G. (2008). Les classes d'objets. Lalies, (28), 111-165.

GROSS, M. (1985). Sur les déterminants dans les expressions figées. Langages, 20(79), 89-117. doi : 10.3406/lgge.1985.2472

GROSS, M. (1988). Les limites de la phrase figée. Langages,23 (90), 7-22. doi : 10.3406/lgge1988.1988

GROSS, M. (1993). Les phrases figées en français. L'information grammaticale, 59(1), 36-41. doi : 10.3406/igram.1993.3139

HULSTIJN, J. H., \& LAUfER, B. (2001). Some empirical evidence for the involvement load hypothesis in vocabulary acquisition. Language Learning, 51(3), 539-558. doi:10.1111/0023-8333.00164 HULSTIJ, J. H., \& MARCHENA, E. (1989). Avoidance. Studies in Second Language Acquisition, 11(03), 241-255. doi:10.1017/s0272263100008123

IRUJO, S. (1986). Don't put your leg in your mouth: Transfer in the acquisition of idioms in a second language. Tesol Quarterly, 20(2), 287-304. doi : 10.2307/3586545

KIM, Y., (2008). The role of task-induced involvement and learner proficiency in L2 vocabulary acquisition. Language Learning, 58(2), 285-325. doi:10.1111/j.1467-9922.2008.00442.x

LAUFER, B., \& HULSTIJN, J. (2001). Incidental vocabulary acquisition in a second language: The construct of task-induced involvement. Applied Linguistics, 22(1), 1-26. doi:10.1093/applin/22.1.1

LAUFER, B., \& ELIASSON, S. (1993). What causes avoidance in L2 learning. Studies in Second Language Acquisition, 15(1), 35-48. doi:10.1017/s0272263100011657

LIAO, Y., \& FUKUYA, Y. J. (2004). Avoidance of phrasal verbs: The case of Chinese learners of English. Language learning, 54(2), 193-226.

MACKEY, A. (2013), Conversational interaction in second language acquisition. Oxford University Press.

MEJRI, S. (2008). Constructions à verbes supports, collocations et locutions verbales. Las construcciones verbo-nominales libres y fijas. Aproximación contrastiva y traductológica, 191-202. 
NATION, I. S. P. (2001). Chunking and collocation. In Learning Vocabulary in Another Language, 317343. doi:10.1017/cbo9781139524759.011

NEWTON, J. (2013). Incidental vocabulary learning in classroom communication tasks. Language Teaching Research, 17(2), 164-187. doi:10.1177/1362168812460814

SIYANOVA, A., \& SCHMITT, N. (2007). Native and nonnative use of multi-word vs. one-word verbs. IRAL-International Review of Applied Linguistics in Language Teaching, 45(2), 119-139. doi : 10.1515/ iral.2007.005

SOLEIMANI, H. (2015). Vocabulary acquisition and task effectiveness in Involvement Load

Hypothesis: A case in Iran. International Journal of Applied Linguistics and English Literature, 4(5). doi:10.7575/aiac.ijalel.v.4n.5p.198

TSUBAKI, M. (2007). The involvement load hypothesis: an inquiry into vocabulary learning. 文京学 院大学外国語学部文京学院短期大学紀要, (6), 175-184.

WRAY, A., \& PERKINS, M. R. (2000). The functions of formulaic language: An integrated model.

Language \& Communication, 20(1), 1-28. doi:10.1016/s0271-5309(99)00015-4

\section{NOTES}

1. De son côté, Nation (2000) souligne que l'utilisation de la langue est colocationnelle ( colocational). Selon cet auteur, les collocations sont des groupes de mots qui apparaissent souvent ensemble.

2. Toujours selon Laufer \& Eliasson (1993), les apprenants d'une L2 adoptent souvent plusieurs stratégies afin d'éviter la forme cible.

3. Tandis que d'autres élaborent des tâches proposées aux apprenants menant à l'acquisition des mots simples où l'implication de l'apprenant est mise en exergue (Garza \& Harris, 2016; Kim, 2008; Soleimani, 2015; Laufer \& Hulstijn, 2001; Hulstijn \&Laufer, 2001; Newton, 2013; Mackey, 2013; Tsubaki, 2007, etc.) Dans ces travaux, les chercheurs analysent l'efficacité de l'hypothèse de la charge d'implication (proposée par Hulstijn \& Laufer, 2001).

4. En ce qui a trait aux verbes complexes en français, l'opacité sémantique est une propriété qui définit généralement les LV et non pas les CAVS (G. Gross, 1996a; Schneider, 1991a).

5. Gonzàlez (2008, 2007), Detry (2008) aussi bien que Irujo (1986) s'accordent à affirmer que l'apprentissage des constructions complexes est facilité si l'apprenant peut se référer aux constructions de sa L1 afin d'en trouver le sens.

6. Nous nous sommes inspirés des tâches proposées par Liao \& Fukuya, 2004.

7. Dans plusieurs phrases de cet exercice, les participants pouvaient trouver comme synonyme soit une LV (dans cet exemple jeter la serviette), soit une CAVS (donner sa démission), soit un autre verbe prédicatif simple (quitter le travail).

8. Tâche qui consistait en la reformulation d'un verbe prédicatif simple.

9. Tâche qui consistait en la traduction d'une LV ou d'une CAVS de l'anglais vers le français.

10. Dans le cadre de cette tâche, les participants pouvaient choisir soit un verbe complexe soit un verbe simple.

11. Ici, les participants pouvaient choisir soit un verbe simple, soit un verbe complexe, soit la proposition que les deux sortes de verbes étaient équivalentes.

12. Par contre, les apprenants du FL2 ne remarquaient pas la synonymie entre les constructions verbales complexes et les verbes simples aussi souvent que les locuteurs natifs de cette langue.

13. Dans une étude plus récente, Gonzales (2014) souligne que les structures figées sont de plus en plus enseignées aux apprenants des L2. 


\section{ABSTRACTS}

The goal of this study is to analyse the differences between the idioms and the light verb constructions in French as perceived by native speakers of this language and by the FL2 learners. We have conceived four tasks that allowed us to analyse many aspects of complex verb mastery by the two groups. This study shows that FL2 learners perceive phrasal verbs at the same rate as the native speakers of that language. Contrariwise, the native speakers produce more often those verbs.

L'objectif de ce travail de recherche est d'étudier la distinction formelle entre les locutions verbales et les constructions à verbe support telles qu'elles sont présentées et décrites par les chercheurs travaillant dans le cadre théorique du lexique-grammaire. Dans ce but, nous avons conçu quatre tâches que nous avons proposées à nos deux groupes de participants : des locuteurs natifs du français et des apprenants du FL2. Nous avons testé plusieurs aspects de la maîtrise des constructions verbales complexes en français par nos participants en mettant en lumière les différences dans les tâches de perception ainsi que dans les tâches de production des structures ciblées. Cette étude démontre que, bien que la perception des structures verbales complexes par les apprenants du FL2 soit assez semblable à celle des locuteurs natifs de cette langue, leur production représente un aspect assez difficile pour les premiers.

\section{INDEX}

Keywords: idioms, light verb construction, phrasal verb, lexique-grammaire, acquisition.

Mots-clés: locution verbale, construction à verbe support, verbes complexe, lexique-grammaire, acquisition

\section{AUTHORS}

\section{ALMA BULUT}

Université Concordia - Montréal (Québec) Canada

ADEL JEBALI

Université Concordia - Montréal (Québec) Canada 